\title{
ENERGY EFFICIENT QUATERNARY CAPACITIVE DAC SWITCHING SCHEME FOR SAR -ADC
}

\author{
Mr. Sarvesh S Chavhan ${ }^{1 *}$, Prof. Mrs. K.M. Bogawar ${ }^{2}$ \\ *1,2Electronics Engineering Department, PCE Nagpur, India \\ ${ }^{* 1}$ Email: chavhansarvesh@gmail.com, ${ }^{2}$ Email: karuved@rediff.com
}

*Corresponding Author: -

Email: chavhansarvesh@gmail.com

\begin{abstract}
: -
This paper presents energy efficient 4-bit successive approximation register analog to digital converter (SAR-ADC) for neural recording front end interface of neural prosthetic system (Brain machine interface). The energy efficient quaternary capacitive switching scheme $(Q C S)$ in the implementation of capacitive digital to analog converter $(C$-DAC) is employed which makes the energy consumption in the C-DAC independent of the output digital code. The proposed quaternary capacitive technique in C-DAC achieves a 50\% reduction in the average energy consumption. The design is implemented in 0.25um standard complementary metal-oxide semiconductor technology (CMOS).
\end{abstract}

Keywords: -Neural System; Brain Machine Interface; Switching Scheme; Capacitive DAC; Quaternary Capacitive Switching Scheme; SAR-Logic Module; Successive Approximation Registers Analog to Digital Converter ( SAR-ADC).

\section{(a) $(\$)$}




\section{INTRODUCTION}

Recording the brain neural activity is important to diagnose the neurological disorders such as epilepsy, depression, and Parkinson's disease. To record these brain neural activities there is great demand for miniature implantable integrated Microsystems called as neural prosthetic brain machine interface. Many previous works have shown progress towards designing these low power Microsystems [1]-[5]. The Fig.1 shows the generalized block diagram of neural prosthetic brain machine interface system.

This neural prosthetic brain machine interface system typically consists of recording and stimulation channel. The stimulation channel concerns with the last requisite of the brain machine interface and help to control the movement of the different body parts. The recording channel concerns with the first requisite of the brain machine interface and commonly known as neural recording front end. The main role of neural recording front end is to sense the neural signals extracted from the number of neurons, amplify, and digitize them without corrupting it with electronic noise. The neural recording front end typically consists of a multi-electrode array (MEA) for capturing the neural activity, followed by a bank of low noise amplifiers (LNAs) to amplify the captured neural signals, and analog to digital converter to digitize the recorded neural information.

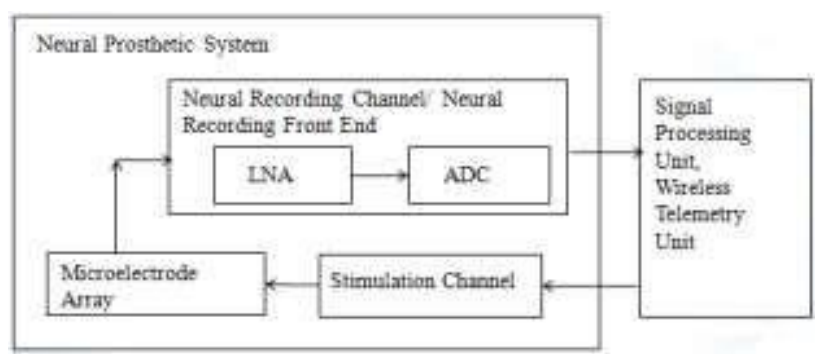

Fig.1. Implantable system for brain machine interface system.

An analog to digital converter is very important block in the neural recording front end design. There has been a lot of work in the designing of an energy efficient analog to digital converter. Motivated by the need for energy efficient solutions for analog to digital converter, in this paper we present a solution for ADC. The use of successive approximation registers (SAR) analog to digital converter (ADC) is preferred for neural recording system design due to moderate resolution (8-10 bits) and high energy efficiency. To implement the SAR-ADC we need the capability to compare the input with a reference levels and control these reference levels based on the result of previous comparison. Fig. 2 shows the block diagram of a conventional SAR-ADC.

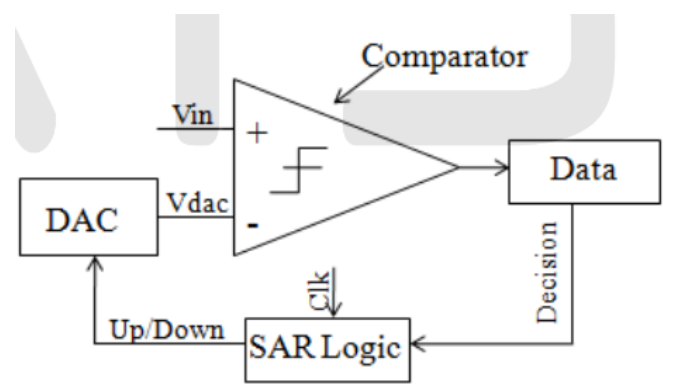

Fig. 2. Block diagram for conventional SAR-ADC.

This paper is organized as follow. Section II presents the proposed architecture for 4-bit SAR-ADC. Section II(A) presents the circuit implementation of comparator. Section II(B) explains the energy efficient QCS-DAC switching technique. Section II(C) discusses of successive approximation register digital logic module implementation. Section III presents the measured simulation results of the comparator.

III.SAR-ADC ARCHITECTURE

Fig. 3 shows the proposed architecture of 4-bit SAR-ADC with quaternary capacitive DAC and flipping mechanism to flip the DAC output for negative difference between two DAC outputs. 


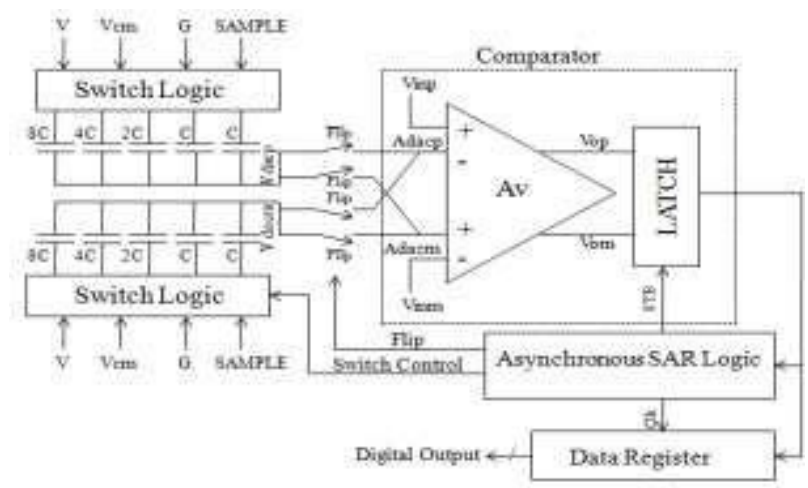

Fig. 3. Proposed architecture of 4-bit SAR-ADC

A comparator is used to compare input Vin with reference level Vdac(Adacp or Adacm). Comparator design is discussed in section-II(A). To generate reference levels digital to analog converter (DAC) is used. Capacitive-DAC(C-DAC) is predominantly employed among various kinds of DACs due to the advantage of no static power dissipation. Energy consumption in the DAC is a significant part of total energy consumption in the ADC and an energy efficient solution of the ADC should try to reduce the energy consumption in the ADC. This paper, presents a novel energy efficient DAC switching technique [6]-[7], quaternary capacitive DAC switching scheme (QCS), which makes energy consumption in DAC small compared to conventional switching scheme, discussed in section-II(B) in detail.

\section{A. Comparator}

The schematic diagram of the comparator is shown in the fig.4. The comparator consists of two blocks, pre-amplifier and latch. The noise and input referred offset are considered as the limiting factors of the resolution, one of the performance metrics of comparator. To mitigate the effect of offset and kickback noise the use of pre-amplifier is preferred. The preamplifier in SAR-ADC amplifies the residual difference between the DAC voltage and input voltage just enough for the detection of the sign by the latch by using the STB signal which is generated in the SAR logic block.

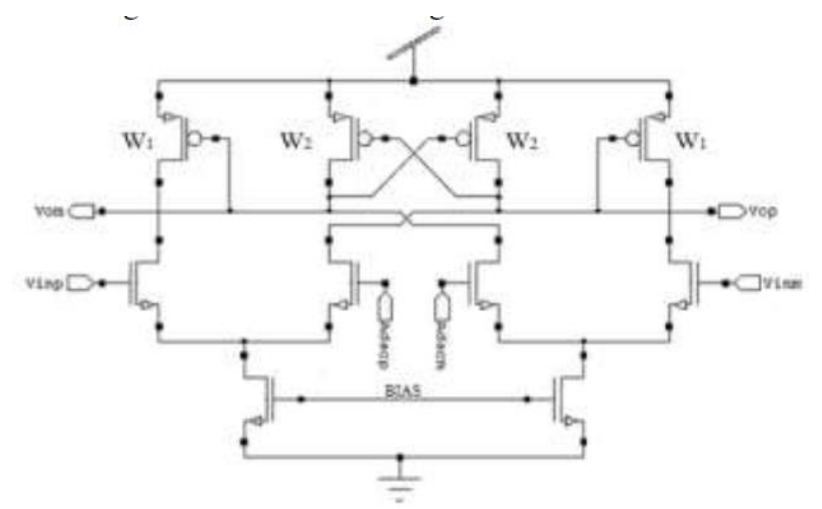

Fig. 4(a). Schematic diagram of Pre-amplifier

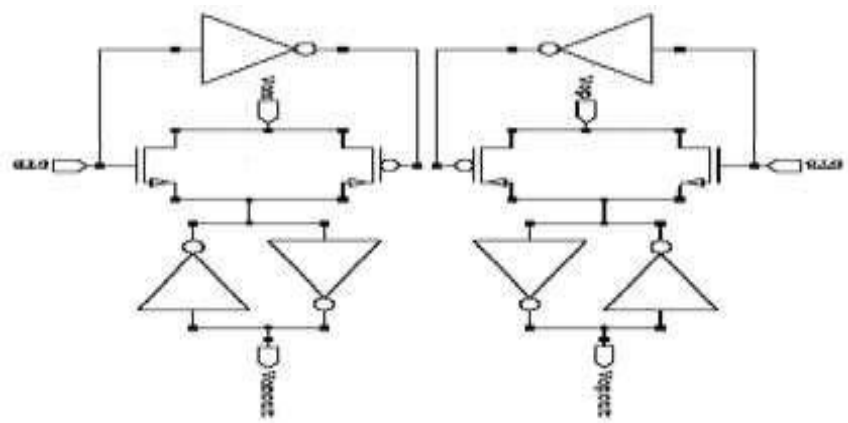

Fig. 4(b). Schematic diagram of simple Latch

The pre-amplifier consists of two pair of transistors each for Vin and Adac. The partial feedback is achieved by transistor of width W2 which reduces the effective output conductance and the speed of operation of the comparator is improved. The width of load transistor (W1) kept greater than the width of the transistor providing partial feedback (W2) to prevent the effective output conductance from becoming negative, even in the presence of mismatch.

\section{B. Quaternary Capacitive Switching Scheme}

The SAR-ADC works on the principle of binary search algorithm. Based on the result of comparison, the binary search algorithm progress to compare higher or lower voltage level. That is it makes up or down transition for the next 
comparison. But it has been shown that, the energy consumption during the down transition is more than up transition [8] during the successive comparison in the implementation of binary search tree.

The energy consumed in down transitions differ the energy consumed during that of up transitions. And it is because of interference of higher MSB capacitors during the extraction of lower significant bits. This makes the energy consumption in the capacitive DAC (C-DAC) dependent on the output code. The MSB capacitors interfere during the extraction of LSBs hence degrades the energy consumption during the down transitions.

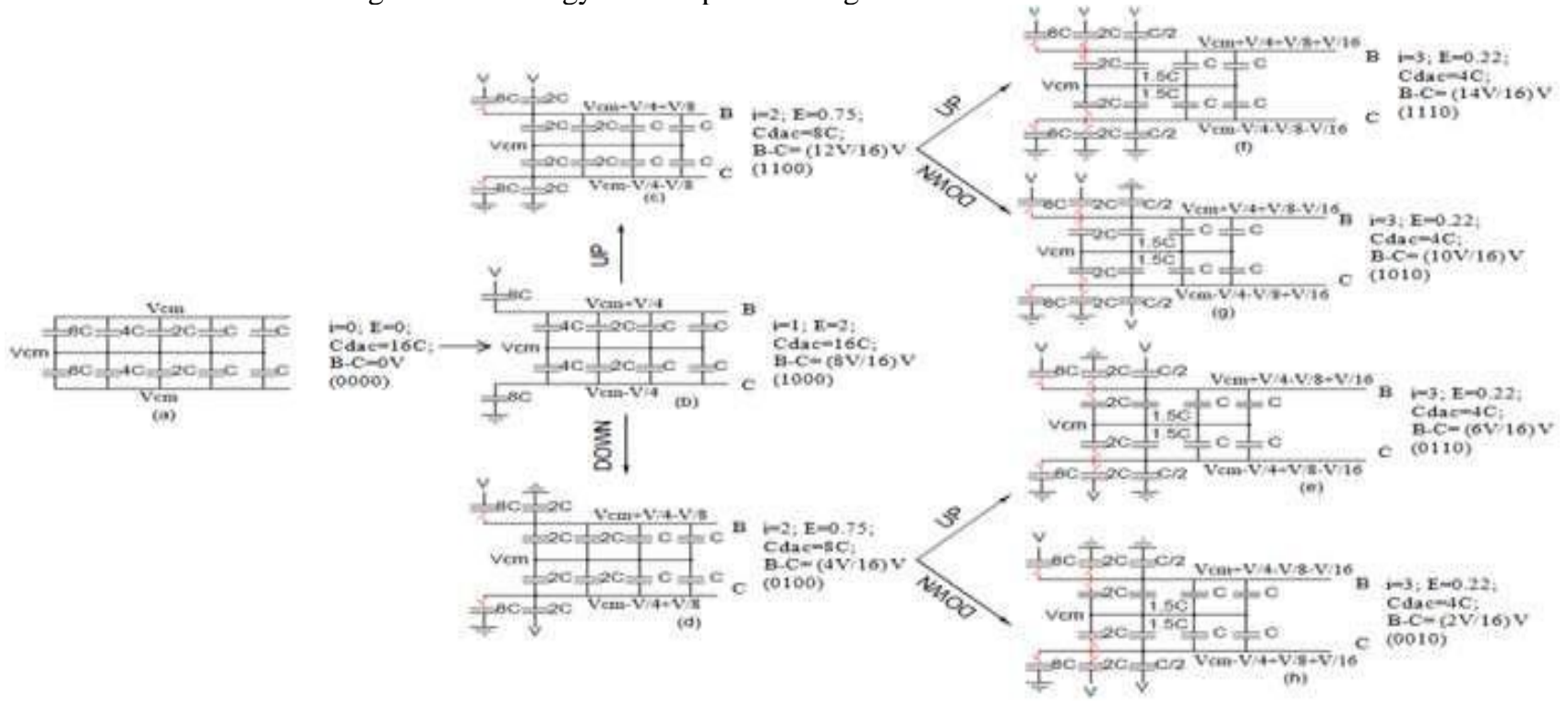

Fig. 5. Proposed switching technique with Quaternary Switching Capacitors

The fig. 5 shows the binary weighted C-DAC switching scheme for a 4-bit SAR-ADC. The figure shows the proposed scheme, in which, after each bit extraction, the effective capacitance reduces by floating the last MSB capacitors. Hence the MSB capacitors do not affect the energy consumption during the extraction of the remaining lower significant bits. The amount of energy delivered by the reference to the C-DAC is then only dependent on the capacitance to be switched from $\mathrm{Vcm}$ to reference voltage level (Vref or V). The proposed energy switching scheme reduces the energy drawn from the reference by reducing the switching capacitance, compared to conventional switching schemes. As the MSB capacitors are not in circuit during the estimation of LSBs, the proposed scheme makes the energy consumption in the CDAC independent of the output digital code.

Fig. (c) and fig. (d) represent up and down steps respectively for the estimation the second MSB. For this, first MSB capacitor $(=8 \mathrm{C})$ is made to float which enables switching of only $2 \mathrm{C}$ for step changes of $\mathrm{V} / 8$ in the CDAC output compared to $4 \mathrm{C}$ in [9]. The energy drawn from the reference is

$3 / 4 . \mathrm{CV}^{2}$ for both up and down transitions in this step. The energy consumption for these steps in [10] $\operatorname{are~Eup=1/2.CV}{ }^{2}$ and Edown $=5 / 2 . \mathrm{CV}^{2}$, i.e. Eavg $=3 / 2 \mathrm{CV}^{2}$. Hence the proposed technique achieves a $50 \%$ reduction in the average energy consumption for this step. Similarly, fig (e-h) shows that floating the $2^{\text {nd }}$ MSB capacitor $(=4 C)$ requires switching of only $0.5 \mathrm{C}$ compared to $2 \mathrm{C}$ in $[9,10]$, for the estimation of third MSB. The size of the switching capacitor for the estimation of $\mathrm{i}^{\text {th }} \mathrm{MSB}$ (excluding sign bit) can be calculated as,

\section{Cswitching $(\mathrm{i})=\mathrm{Cdac} / 4^{\mathrm{i}}$}

Where Cdac is the total DAC capacitance and is equal to $2^{\mathrm{n}} \mathrm{C}$ for an n-bit DAC. The size of switching capacitor reduces in a quaternary fashion in the proposed scheme.

During the extraction of LSBs, the MSB capacitors are removed, and the removal of MSB capacitors create a net residual charge on the $\mathrm{C}$-DAC output rail which preserves the previous value of the C-DAC output. Hence the switching of the LSB capacitors needs to do only the incremental work. Also, in this scheme only one set of capacitors is switched at a time, $\mathrm{Vcm}$ to reference voltage level or ground $\mathrm{G}$, which keeps the design of the digital logic simple and energy efficient. The average energy consumption for an n-bit ADC for the proposed switching scheme can be calculated using the expression,

$$
\operatorname{Eavg}(n)=\sum_{i=1}^{n-1} 2^{n-x-1} \cdot\left(1-\frac{1}{z^{2}}\right) C V^{2}
$$

As the higher MSB capacitors do not affect the energy consumption during the estimation of LSBs, the energy consumed for up and down transitions are always equal which makes the energy consumption independent of the output code. Also, due to reduction in the size of the switching capacitance it is to achieve a faster settling in the DAC and hence improves 
the speed of the successive approximation register (SAR) ADC.

If the DAC voltage is negative, then its sign can be converted by either interchanging the two DAC inputs to the comparator or by comparing this negative DAC voltage with negative of the sampled input voltage. The interchanging of two DAC inputs to the comparator is preferred as the latter approach will flip the offset of the comparator which can affect the linearity of the ADC. To flip these voltages, it does not take any extra clock cycle and hence speed is not compromised.

\section{SAR Logic Module}

SAR-ADC implements the binary search algorithm using the SAR control logic. Successive approximation register (SAR) control logic determines each bit successively or sequentially based on the result of the comparator. The control logic uses a ring counter and a code register. The ring counter is basically a shift register.

In each clock cycle, one of the outputs in the ring counter sets a flip flop in the code register. The output of the flip flop which is set by the ring counter is used as the clock signal for the previous flip flop. At the rising edge of the clock this flip flop loads the result from the comparator. At the end of each conversion, EOC signal is issued to halt the conversion. For low power purpose, transmission gate based set reset D-flip flops are used.

\section{IV.EXPERIMENTAL RESULTS}

The fig. 6(a) and fig. 6(b) shows the magnitude and phase response of the preamplifier. The simulated values are $A v=22$ $\mathrm{dB}$ and $\mathrm{Wp}=30 \mathrm{KHz}$. Fig. (c) shows the transient response of the comparator.

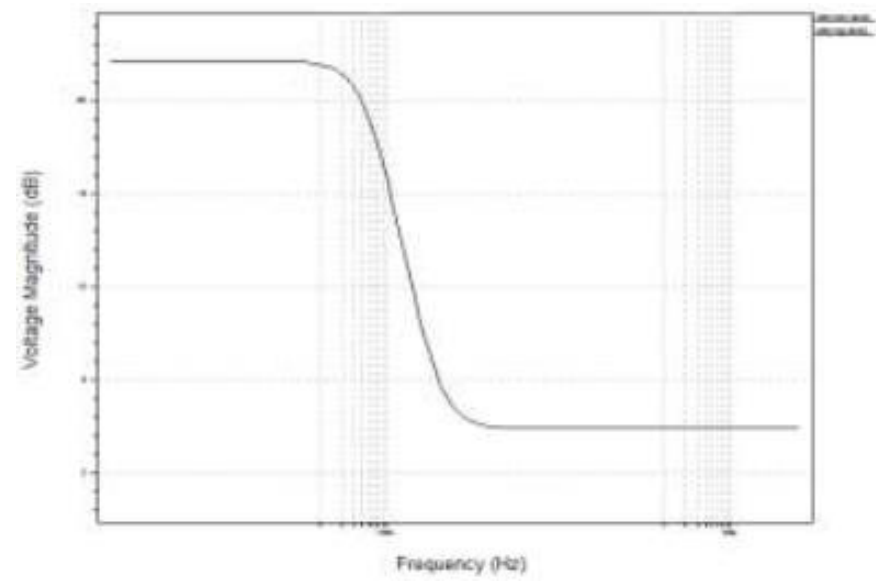

Fig. 6(a). Magnitude Response of Preamplifier

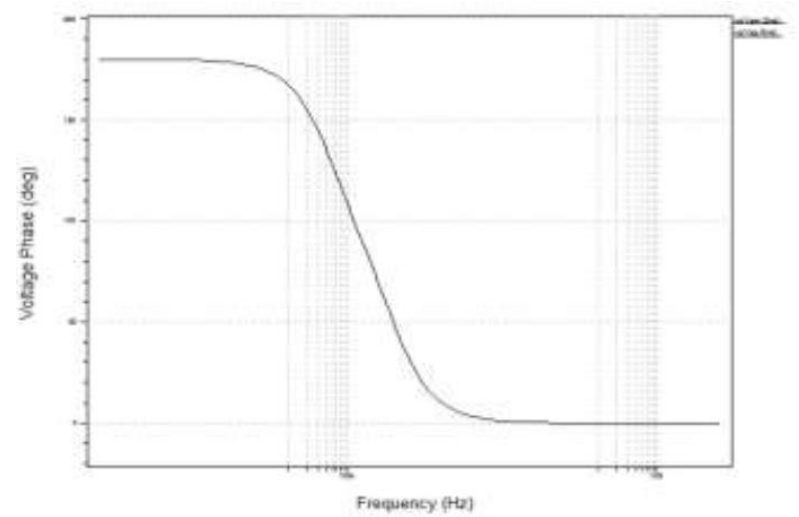

Fig. 6(b). Phase Response of Preamplifier 


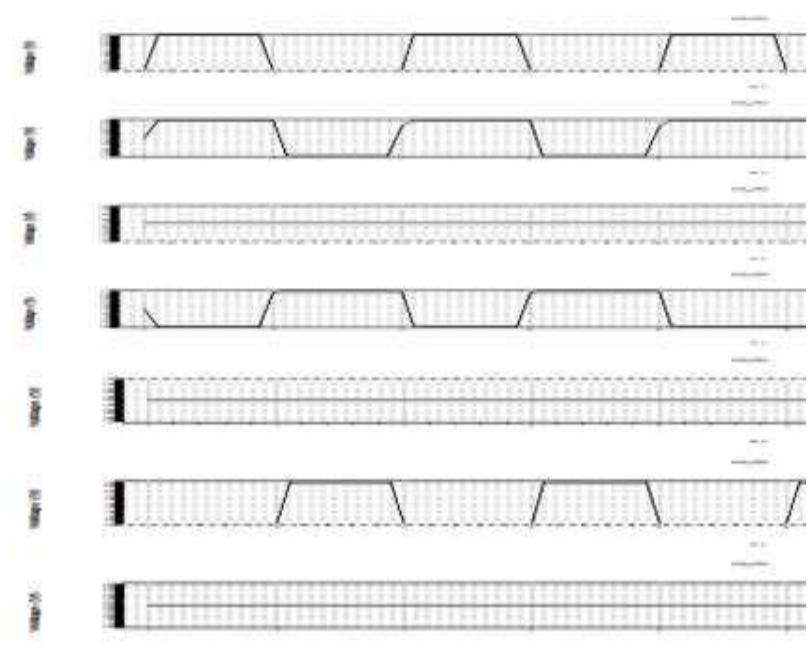

Fig. 6(c). Transient Response of Preamplifier

\section{REFERENCES}

[1].K. Chen, Z. Yang, L. Hoang, J. Weiland, M. Humayun, and W. Liu, "An Integrated 256-Channel Epiretinal Prosthesis," Solid-State Circuits, IEEE Journalof, vol. 45, no. 9, pp. 1946 -1956, sept. 2010.

[2].J. Fayad, S. Otto, R. Shannon, and D. Brackmann, "Cochlear and BrainstemAuditory Prostheses" Neural Interface for Hearing Restoration: Cochlear and Brain Stem Implants"," Proceedings of the IEEE, vol. 96, no. 7, pp. 1085 -1095, july 2008.

[3].J. He, C. Ma, and R. Herman, "Engineering Neural Interfaces for Rehabilitationof Lower Limb Function in Spinal Cord Injured," Proceedings of the IEEE, vol. 96, no. 7, pp. $1152-1166$, july 2008.

[4].M. Liker, D. Won, V. Rao, and S. Hua, "Deep Brain Stimulation: An Evolving Technology," Proceedings of the IEEE, vol. 96, no. 7, pp. $1129-1141$, july 2008.

[5].A. Amar, M. Levy, C. Liu, and M. Apuzzo, "Vagus Nerve Stimulation,” Pro- ceedings of the IEEE, vol. 96, no. 7, pp. $1142-1151$, july 2008.

[6].M. Azin, D. Guggenmos, S. Barbay, R. Nudo, and P. Mohseni, “A batterypowered activity-dependent intracortical microstimulation IC for brain machine- brain interface," Solid-State Circuits, IEEE Journal of, vol. 46, no. 4, pp. 731 -745 , april 2011.

[7].U. Frey, F. Heer, R. Pedron, S. Hafizovic, F. Greve, J. Sedivy, K.-U. Kirstein,and A. Hierlemann, "An 11k-electrode 126-channel high- density microelectrode array to interact with electrogenic cells," in Solid-State Circuits Conference, 2007. ISSCC 2007. Digest of Technical Papers. IEEE International, feb. 2007, pp. 158 -593.

[8].B. Ginsburg and A. Chandrakasan, "An energy-efficient charge recycling approachfor a SAR converter with capacitive DAC," in Circuits and Systems, 2005. ISCAS 2005. IEEE International Symposium on, may 2005, pp. 184 -187 Vol. 1.

[9].Y. Zhu, C.-H. Chan, U.-F. Chio, S.-W. Sin, S.-P. U, R.Martins, and F.Maloberti, “A 10-bit 100-MS/s reference-free SAR ADC in 90 nm CMOS,” Solid-State Circuits, IEEE Journal of, vol. 45, no. 6, pp. 1111 - 1121, june 2010.

[10]. T. Anand, V. Chaturvedi, and B. Amrutur, "Energy efficient asymmetric binary search switching technique for SAR ADC," Electronics Letters, vol. 46, no. 22, pp. 1487 -1488, 282010.

[11]. Vikram Chaturvedi, Tejasvi Anand, and Bharadwaj Amrutur, "An 8-to-1 bit 1-MS/s SAR ADC With VGA and Integrated Data Compression for Neural Recording," IEEE Transactions on Very Large-Scale Integration (VLSI), vol. 21, no. 11, November 2013.

[12]. Farzaneh Shahrokhi, Karim Abdelhalim, Demitre Serletis, Peter L. Carlen, and Roman Genov, "The 128-Channel Fully Differential Digital Integrated Neural Recording and Stimulation Interface," IEEE Transaction on Biomedical circuits and System, vol. 4, no. 3, June 2010. 